Check for updates

Cite this: RSC Adv., 2017, 7, 41077

\title{
First-principles study on the electric structure and ferroelectricity in epitaxial $\mathrm{CsSnl}_{3}$ films $^{\dagger}$
}

\author{
Guang Song, (D) *ab Benling Gao, ${ }^{a}$ Guannan Lic ${ }^{\mathrm{ac}}$ and Jun Zhang ${ }^{\mathrm{a}}$
}

Ferroelectricity is a potentially crucial issue in inorganic halide perovskites, which are breakthrough materials in photovoltaic research. However, to date, conclusive evidence for ferroelectricity in allinorganic halide perovskites is still lacking. Herein, using density functional theory simulations and symmetry analysis, it is found that two stable ferroelectric phases, $P 4 \mathrm{bm}$ and $P m c 2_{1}-(I)$, can be induced by compressive and tensile strain in all-inorganic halide perovskite $\mathrm{CsSnl}_{3}$ films. More importantly, the calculated polarization value is as high as several $\mu \mathrm{C} \mathrm{cm}^{-2}$ which is desirable for the separation of photo-excited carriers in these materials. In addition, it is found that the P4bm phase has an indirect band gap and the $P m c 2_{1}-(I)$ phase has a suitable direct band gap for the absorption of visible light. In particular, tensile strain can alter the band gaps by several tenths of an electronvolt in the $\operatorname{Pmc}_{1}-(\mathrm{I})$ phase. Overall, these results give useful insight for strained $\mathrm{CsSnl}_{3}$ films and hopefully provide a new route to design ferroelectric semiconductors for photovoltaic materials in the less-explored inorganic halide perovskites.

Received 14th July 2017

Accepted 7th August 2017

DOI: $10.1039 / \mathrm{c} 7 \mathrm{ra07735a}$

rsc.li/rsc-advances near the Fermi level, which increases the opportunity for electron-hole recombination during carrier transport from the bulk to the surface of the material. ${ }^{10}$ In this context, designing smaller band gap FE materials with long carrier diffusion lengths is highly desired; however, this task is conceptually challenging.

Organic-inorganic hybrid perovskites, most notably methylammonium lead triiodide $\left(\mathrm{MAPbI}_{3}\right)$, exhibit semiconducting band gaps suitable for the absorption of visible light, long range carrier diffusion lengths, and weak exciton binding energies, and thus, have made a breakthrough in the photovoltaic field. ${ }^{\mathbf{1 1 1 - 1 5}}$ In addition to its preeminent semiconducting merits, large FE polarization $\left(\sim 38 \mu \mathrm{C} \mathrm{cm}^{-2}\right)$ is estimated in $\mathrm{MAPbI}_{3}$ by comprehensive calculations. ${ }^{\mathbf{1 6}, 17}$ However, due to the complex dynamic motions of organic $\mathrm{MAPbI}_{3}$, conclusive evidence is still lacking for the bulk ferroelectricity of $\mathrm{MAPbI}_{3}$, such as dielectric anomaly and electric hysteresis loops. ${ }^{18}$ Recently, another organic-inorganic hybrid perovskite, $\mathrm{CH}_{3} \mathrm{NH}_{3} \mathrm{GeI}_{3}$, has attracted much attention due to its lead-free and highly distorted structure. In a previous study, a detailed theoretical investigation was conducted to predict the total FE polarization, which was about $13.8 \mu \mathrm{C} \mathrm{cm}{ }^{-2} \cdot{ }^{19}$ Despite the enormous success of organic-inorganic hybrid halide perovskites in solar cell research, a serious issue is their poor long-term device stability, especially under heat and humidity conditions, in which hinders their large-scale commercial applications. To resolve this issue, the use of inorganic cations such as $\mathrm{Cs}^{+}$to replace $\mathrm{CH}_{3} \mathrm{NH}_{3}{ }^{+}$at the A site (e.g. $\mathrm{CsPbCl}_{3}, \mathrm{CsPbBr}_{3}$, and $\mathrm{CsPbI}_{3}$ ) significantly increases the stabilities of halide perovskites. ${ }^{\mathbf{1 2 , 2 0 - 2 3}}$ However, investigations on the ferroelectrics of all-inorganic
${ }^{a}$ Department of Physics, Huaiyin Institute of Technology, Huaian 223003, China. E-mail: gsong1840@gmail.com

Jjiangsu Provincial Key Laboratory of Palygorskite Science and Applied Technology, Huaiyin Institute of Technology, Huaian 223003, China

${ }^{c}$ National Laboratory of Solid State Microstructures, Department of Physics, Nanjing University, Nanjing 210093, China

$\dagger$ Electronic supplementary information (ESI) available. See DOI: 10.1039/c7ra07735a 
halide perovskites are scarce. Consequently, it is of great interest to induce ferroelectricity in halide perovskites consisting of totally inorganic components having good stability and possessing suitable band gaps for absorption in the solar spectrum.

To induce the ferroelectricity in inorganic halide perovskites, a straightforward method is to follow the ideas used for perovskite oxides. A typical example is the hybrid improper FE mechanism which provides a robust route to achieve ferroelectricity from nonpolar components. ${ }^{24-27}$ In this regard, previous study has predicted that the inversion symmetry in $\mathrm{CsSnI}_{3}$ can be broken by ordering $\mathrm{Cs}^{+}$by other cations (e.g. $\left.\mathrm{Rb}^{+}\right)$ at the atomic scale on the perovskite A site. In this system, the calculated band gap is $1.58 \mathrm{eV}$ which is suitable for absorption in the solar spectrum. Nevertheless, the calculated electric polarization is about $1 \mu \mathrm{C} \mathrm{cm}{ }^{-2}$ which is smaller than that for organic-inorganic hybrid halide perovskites. ${ }^{28}$ In this context, designing FE inorganic perovskite halides with larger electric polarization is still highly desirable.

The central objective of this study is to search for allinorganic FE halide perovskites possessing suitable band gaps and large electric polarization. With first-principle calculations, we comprehensively investigate the effects of biaxial strain and the accompanying structural distortions on the energy landscapes, electric polarization, and band gaps of films of the perovskite photovoltaic material $\mathrm{CsSnI}_{3}$, which is a counterpart of full-inorganic perovskite. $\mathrm{CsSnI}_{3}$ exhibits semiconducting and high carrier mobilities. ${ }^{29,30}$ Moreover, $\mathrm{CsSnI}_{3}$ perovskite photovoltaic devices exhibit stability $\sim 10$ times greater than devices with the same architecture using $\mathrm{MAPbI}_{3} \cdot{ }^{31}$ Herein, it is found that both compressive and tensile strain can drive the non-polar phase (Pnma) into the polar phase $(P 4 b m$ in the compressive range and $P m c 2_{1}$-(I) in the tensile range). Emphatically, our calculations show that the FE polarization is up to several $\mu \mathrm{C} \mathrm{cm}^{-2}$ and the polarization is greatly enhanced compared with a previous study. Moreover, we show that compressive strain can induce a direct-indirect band gap transformation accompanied with a Pnma to P4bm transition. The $P m c 2_{1}$-(I) phase has a suitable direct band gap for the absorption of visible light and tensile strain can alter the band gaps by several tenths of an electronvolt in this phase. In addition, we also predict that there is an electric-elastic response in the vicinity of the phase boundary of the $P m c 2_{1}$-(I) and $P m c 2_{1}$-(II) phases. Therefore, it is expected that the strained $\mathrm{CsSnI}_{3}$ films can serve as light-absorbing materials and these results can stimulate the study of ferroelectricity in lessexplored inorganic halide perovskites.

\section{Computational methods}

Optimization for crystal structures and computations on electronic properties were based on density functional theory (DFT) within the PBEsol exchange correlation functional ${ }^{32}$ implemented in the VASP package. ${ }^{33}$ The electrons taken to be valence were $5 s^{2} 5 p^{6} 6 s^{2}$ of Cs and $5 s^{2} 5 p^{2}$ of $S n$, and $5 s^{2} 5 p^{5}$ of I. A planewave basis set within the projector-augmented-wave method with a $600 \mathrm{eV}$ plane-wave energy cutoff was used throughout. ${ }^{34} \mathrm{~A}$
$\Gamma$-centered $8 \times 8 \times 6 k$-point mesh sampling was used for the $\sqrt{ } 2 \times \sqrt{ } 2 \times 2$ (20 atoms) unit cell. We carefully checked convergence with these settings by testing higher energy cutoffs and larger $k$ meshes. Each self-consistent electronic calculation was converged to $10^{-6} \mathrm{eV}$ and structural relaxations were carried out until the Hellmann-Feynman force was less than $0.005 \mathrm{eV} \AA^{-1}$. According to previous study on related systems, ${ }^{35}$ biaxial strain was modeled using periodic calculations in which in-plane lattice parameters were fixed at lengths ranging from $-9.93 \%$ to $+10.85 \%$ of the respective cubic perovskite lattice parameters $\left(a_{\text {eq }}=8.66 \AA\right)$, while the perpendicular axis and atomic positions were allowed to relax. The electronic contribution to the polarization was calculated following the Berry phase formalism. ${ }^{36}$ Space groups were determined using the FINDSYM software. ${ }^{37}$ We also examined the electronic structures for VASP-predicted perovskite iodide structures using the HSE06 (Heyd-Scuseria-Ernzerhof) hybrid functional. ${ }^{38}$ Structural images were produced using the VESTA software. ${ }^{39}$

\section{Results and discussion}

$\mathrm{CsSnI}_{3}$ forms the cubic perovskite structure above $426 \mathrm{~K}$, tetragonal perovskite structure with the $\mathrm{SnI}_{6}$ octahedral rotation along the $c$-axis between 351 and $426 \mathrm{~K}$, and orthorhombic perovskite structure with additional octahedral tilting below $351 \mathrm{~K} .^{30}$ We firstly optimized the experimentally observed phases of $\mathrm{CsSnI}_{3}$ displayed in Fig. S1.† The calculated and experimental unit cell parameters of bulk $\mathrm{CsSnI}_{3}$ are shown in Table S1. $\dagger$ It can be seen that the calculated lattice constants of the different phases are in good agreement with the experimental value (in all case less than 3\%) and previous theoretical reports. ${ }^{30,35,40}$ Moreover, the energetic preference for the orthorhombic phase is consistent with the fact that this phase is observed at low temperature. All these preparations guaranteed the reliability of the following calculations on strained $\mathrm{CsSnI}_{3}$ films.

Then, we investigated how biaxial strain interacts with the structural energetics of these phases. To explore the phase diagram of the strained $\mathrm{CsSnI}_{3}$ films, the possible crystal structures with different oxygen octahedral tilting patterns, such as antiferrodistortive (AFD), were derived from the unstable phonon modes of the high symmetry $P m \overline{3} m$ structure and their relative stabilities were analyzed. The calculated structures used herein are listed in Table S2. $\dagger$ The total energies per unit cell of the $\mathrm{CsSnI}_{3}$ films as a function of strain are shown in Fig. 1. Despite considering the effect of the epitaxial strain $(e)$ on the different phases with different oxygen octahedral tilting, the total energy of these structures, which are larger than the value of the structures presented in Fig. 1, are not shown. Thus, we only discuss the relatively low energy phases in this study.

As the strain varies from compression to tension, the ground state of the $\mathrm{CsSnI}_{3}$ films changes consecutively from the polar $P 4 \mathrm{bm}$ state to the non-polar Pnma state, then to the polar Pmc2 ${ }_{1}$ (I) state, followed by another polar Pmc2 ${ }_{1}$-(II) state. The structures of these phases are displayed in Fig. 2. Fig. 2a displays the structure of the $P 4 \mathrm{bm}$ state in which the Glazer notation is $\mathrm{a}^{0} \mathrm{a}^{0} \mathrm{c}^{+} .^{41}$ The $\mathrm{SnI}_{6}$ octahedra rotate along the [001] direction in- 


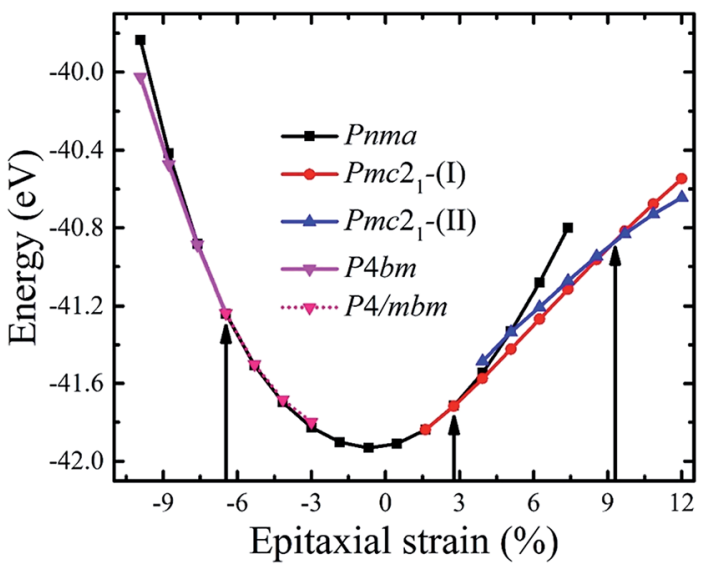

Fig. 1 Computed total energies of the $\mathrm{CsSn}_{3}$ films as a function of biaxial strain perpendicular to the [001] direction with geometries optimized to allow various distortions. The vertical lines mark the phase boundaries between different symmetries. At $e=-6.49 \%$, the $P 4 \mathrm{bm}$ phase transforms into the $P 4 / \mathrm{mbm}$ phase, as indicated by the pink short dotted line, though a second quantum order phase transition.

phase $\left(\mathrm{M}_{2}^{+}\right.$mode, Fig. $\mathrm{S} 2, \dagger$ called $\left.\mathrm{AFD}_{z \mathrm{i}}\right)$. Besides the $\mathrm{AFD}_{z \mathrm{i}}$, there is also an FE mode $\left(\Gamma_{3}{ }^{-}\right.$, called $\left.\mathrm{FE}_{z}\right)$ in the $P 4 b m$ state. Fig. $2 \mathrm{~b}$ displays the structure of the Pnma state which not only includes the $\mathrm{AFD}_{z \mathrm{i}}$, but also $\mathrm{AFD}_{x y}$ (tilts around an axis perpendicular to the (001), $\mathrm{M}_{5}{ }^{-}$mode, Fig. S2 $\dagger$ ), and its Glazer notion is $\mathrm{a}^{-} \mathrm{a}^{-} \mathrm{c}^{+}$. From the structure of $\mathrm{Pmc2}_{1}$-(I) displayed in Fig. 2c, it can be seen that the rotations/tilts of the octahedra in this phase are similar with the Pnma state. Compared with the Pnma state, an $\mathrm{FE}_{x y}$ mode exists in the $P m c 2_{1}$-(I) phase. Fig. $2 \mathrm{~d}$ displays the structure of another $P m c 2_{1}$ phase. Although it has same space group with the aforementioned $P m c 2_{1}$-(I) state, both of them have the $\mathrm{FE}_{x y}$ and $\mathrm{AFD}_{z \mathrm{i}}$ modes; however, the difference between $P m c 2_{1}$-(I) and $P m c 2_{1}{ }^{-}$(II) is that there is no $\mathrm{AFD}_{x y}$ mode in the $P m c 2_{1}$-(II) phase. It is worth mentioning that compared with a previous study on the strain effect on $\mathrm{CsSnI}_{3}$ films, our study is more systematic. ${ }^{35}$ In our study, we not only consider a single factor such as octahedral rotation/tilt or off-center of the Sn cation, but also consider a combination of rotation/tilt and off-center. Therefore, we discovered the polar phase $P 4 \mathrm{bm}$ (compressive) and $\mathrm{Pmc2}_{1}$-(I) (tensile) states. Thus, it is hoped that ferroelectricity can be found in other halide perovskites, such as $\mathrm{CsBX}_{3}(\mathrm{~B}=\mathrm{Ge}, \mathrm{Sn}$ and $\mathrm{Pb} ; \mathrm{X}=\mathrm{Cl}, \mathrm{Br}$ and $\mathrm{I})$ under epitaxial strain.

For the polar $P 4 \mathrm{bm}$ state, its ground state occurs when $e<$ $-6.47 \%$. It should be noted that the $P 4 b m$ state will end up at the $P 4 / \mathrm{mbm}$ state when $e$ is larger than $-6.47 \%$ and this phase transformation is a second order quantum phase transition. First, taking the $P 4 / \mathrm{mbm}$ phase as the reference state, we calculated the electric polarization of this polar state. The calculated results are displayed in Fig. 3a, which shows that the calculated polarization monotonously decreases from 8.25 to $0.17 \mu \mathrm{C} \mathrm{cm}^{-2}$ as strain increases from $-9.93 \%$ to $-6.47 \%$. Obviously, compared with a previous report on the inorganic perovskite (CsRb) $\mathrm{Sn}_{2} \mathrm{I}_{6}$, the calculated polarization is greatly enhanced..$^{28}$ Especially, when strain is larger than $-6.47 \%$, the calculated value of polarization is close to zero. This confirms that the phase transition between the $P 4 \mathrm{bm}$ and $P 4 / \mathrm{mbm}$ phases is a second order transition. We then studied the electric structure of this phase. In Fig. $3 \mathrm{~b}$, the band gaps of the $P 4 \mathrm{bm}$ state are plotted as a function of the epitaxial strain, and to help understand the evolution of the electric structure of $P 4 \mathrm{bm}$ state, the results of the $P 4 / \mathrm{mbm}$ phase are also shown when $e>$ $-6.47 \%$ although it is not the ground state. In particular, the calculated band gaps first decrease from 0.52 to $0.00 \mathrm{eV}$ $(-9.93 \%>e>-5.31 \%)$, and then increase from 0.00 to $0.21 \mathrm{eV}$ $(-5.31 \%>e>-0.69 \%)$. Typically, standard exchangecorrelation functionals such as GGA and LDA significantly underestimate the band gaps. ${ }^{42}$ Due to the importance of iodides in photovoltaic applications, it is essential to compute the electric structure of the strained $\mathrm{CsSnI}_{3}$ films as accurately

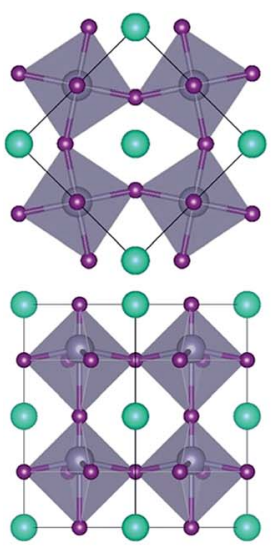

(a)

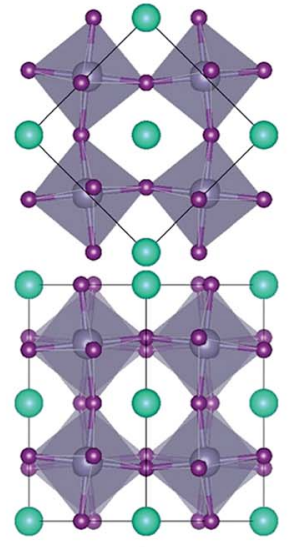

(b)

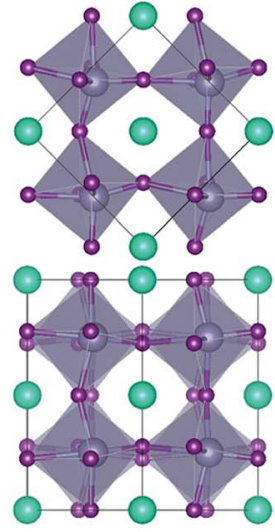

(c)

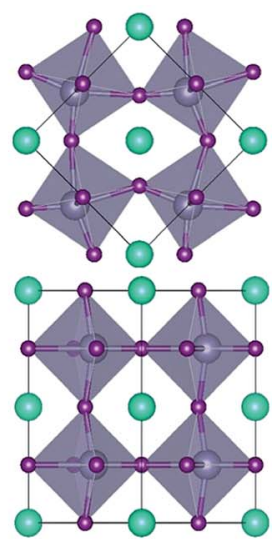

(d)

Fig. 2 Model of the different epitaxial structures. Panels (a), (b) (c), and (d) display a schematic description of the P4bm, Pnma, Pmc2 ${ }_{1}$-(I), and $P m c 2_{1}$-(II) phases, respectively. The first layer represents polyhedral views of these phases from the $c$-axis, while the second layer is the view from the $a$ and $b$-axes. The cyan, gray, and purple balls represent $\mathrm{Cs}$, Sn and I atoms, respectively. 

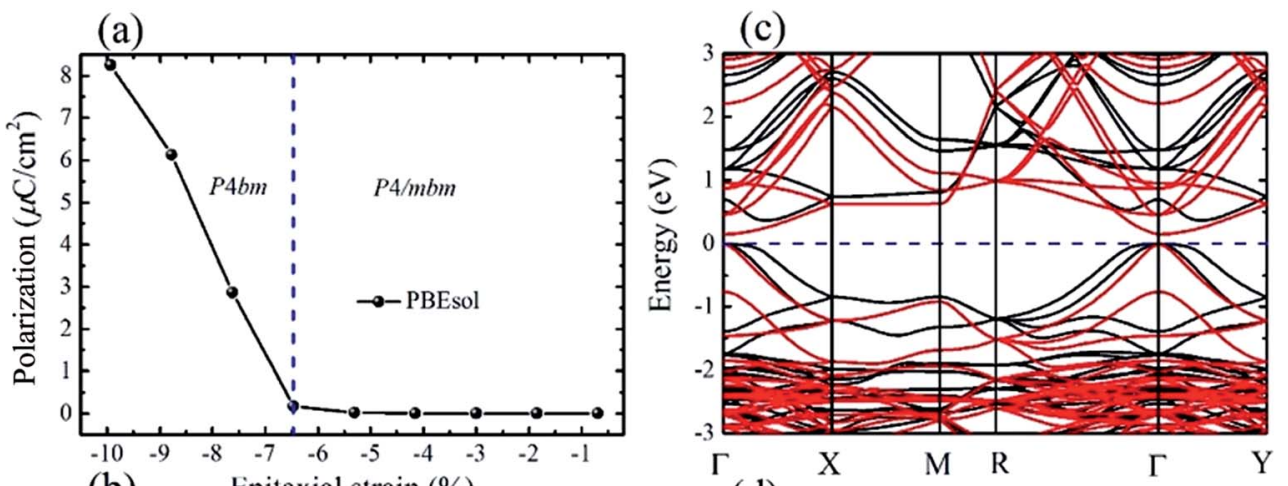

(b)

Epitaxial strain $(\%)$
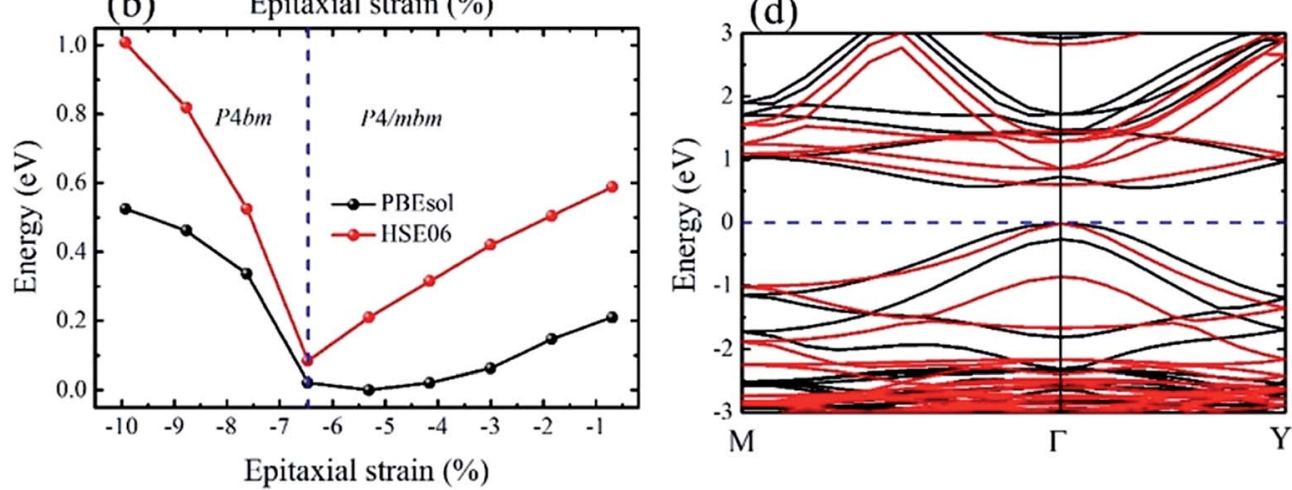

Fig. 3 Electric polarization and electronic properties of the P4bm phase versus epitaxial strain. (a) Electric polarization. (b) Band gaps. (c and d) Band structures of the P4bm and P4/mbm phases calculated using the PBEsol and HSE06 functionals, respectively. The blue dashed lines in (a) and (b) represent the phase boundary of the $P 4 \mathrm{bm}$ and $P 4 / \mathrm{mbm}$ phases. The black lines in (c) and (d) represent the band structure of the $P 4 \mathrm{bm}$ phase at $e=-7.62 \%$, while the red lines represent the $P 4 / \mathrm{mbm}$ phase at $e=-1.85 \%$. The Fermi level is set at $0 \mathrm{eV}$ and is indicated by the blue dashed line.

as possible, especially the energy band gaps. Thus, the HSE06 functional was also selected in these calculations. The calculated results are displayed in Fig. 3b. The calculated band gaps increase compared with that from the PBEsol method and they have a similar trend $v s$. strain increasing. The semiconducting band gaps indicate that this $\mathrm{FE}$ state is not suitable for absorption in the solar spectrum. In addition, we plotted the band-structure of the $P 4 \mathrm{bm}$ state at $e=-7.62 \%$, and the results are displayed in Fig. 3c (PBEsol method) and Fig. 3d (HSE06 functional). It can clearly be seen that in the valence band of the $P 4 b m$ state, the valence band maximum (VBM) is located at the $\Gamma$ point of the Brillouin zone, while the conduction band minimum (CBM) does not lie at $\Gamma$ point, thus, it possesses indirect band gaps. Indirect band gaps are unfavorable for light absorption. Nevertheless, Yu and Zunger previously highlighted an indirect band gap that did not result in weak absorption, and provided a direct allowed transition existing only at higher energy. ${ }^{43}$ Interestingly, after carefully studying the character of this band-structure, we find that there is band inversion (see Fig. S3 $\dagger$ ) at the $\Gamma$ point, which has been reported in topological insulators. Such phenomenon has been discussed in previous reports on other halide perovskites. ${ }^{\mathbf{4 4 , 4 5}}$ Thus, this property allows compressive $\mathrm{CsSnI}_{3}$ thin films to have potential application in the search for new bulk topological insulators.

We now focus on the strain $e$ lying in the region between $-0.47 \%$ and $+1.62 \%$. In this range, the computed ground state is the Pnma state (see Fig. 2). This state is centrosymmetrical in structure, thus it does not possess electric polarization. As the strain increases from $-6.47 \%$ to $+1.62 \%$, the band gaps change from 0.00 to $0.42 \mathrm{eV}$ within the PBEsol method (0.15 to $0.84 \mathrm{eV}$ within the HSE06 method, see Fig. S4†). Moreover, there is a kink in the band gaps curves at $e=-0.69 \%$, because the epitaxial strain breaks the 3-fold degeneracy of the I-5p states, which has been discussed in a previous report. ${ }^{45}$ Similarly to the bulk state, both the VBM and CBM are located at the $\Gamma$ point of the Brillouin zone, thus direct band gaps exist (Fig. S4 $\dagger$ ).

In the region of $+1.67 \%<e<+9.30 \%$, a polar phase named $\mathrm{Pmc2}_{1}$-(I), which takes the place of the Pnma state, becomes the ground state. This phase has been also reported in other perovskite oxide compounds under tensile strain, such as $\mathrm{BiFeO}_{3}$ and $\mathrm{SrZrO}_{3}{ }^{{ }^{\mathbf{4 6}, 47}}$ It is notable that the phase transition between the Pnma and $P m c 2_{1}$-(I) states is also a second order quantum transformation which is similar with the phase transition between the $P 4 \mathrm{bm}$ and $P 4 / \mathrm{mbm}$ phases. The calculated polarization of the strained $\mathrm{CsSnI}_{3}$ films is displayed in Fig. 4a. It can be seen that as the tensile strain increases from $+1.61 \%$ to $+9.30 \%$, the polarization increases monotonically from 0.00 to $13.60 \mu \mathrm{C} \mathrm{cm}^{-2}$. The polarization value is significantly enhanced compared with that in previous reports on inorganic perovskite ferroelectrics and is the same order of magnitude with organic halide perovskite ferroelectrics such as $\mathrm{CH}_{3} \mathrm{NH}_{3} \mathrm{GeI}_{3} \cdot{ }^{19,28}$ To confirm that the ferroelectricity of the $\mathrm{Pmc2}_{1}$-(I) phase can be 

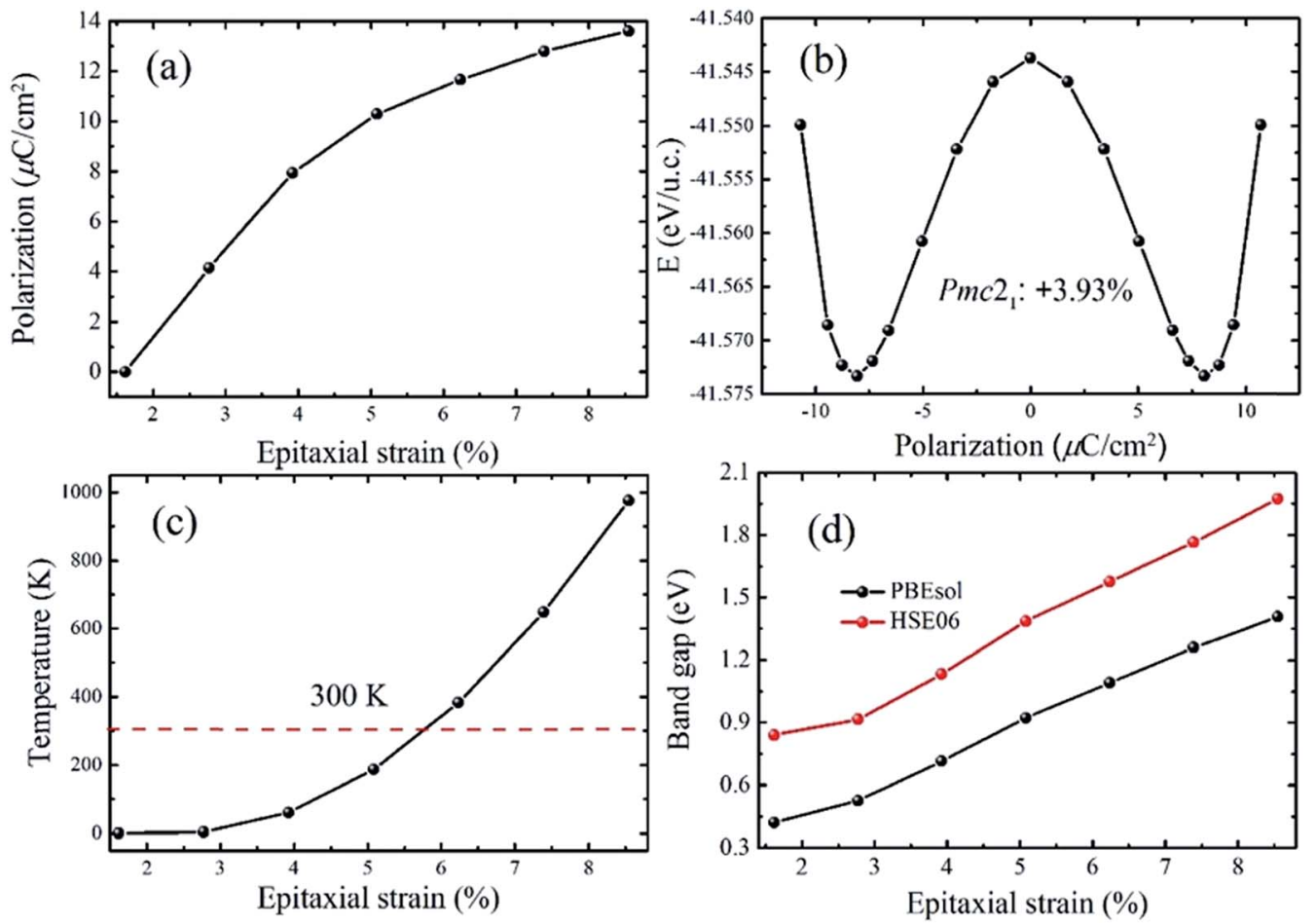

Fig. 4 Electric polarization and electronic properties of the $P m c 2_{1}-$ (I) phase versus epitaxial strain. (a) Electric polarization. (b) Double well of the $P m c 2_{1}$-(I) phase at $e=+3.93 \%$. (c) Estimated Curie temperature of the Pmc2 ${ }_{1}$-(I) phase. The red dashed line indicates $300 \mathrm{~K}$. (d) Band gaps.

switched by an epitaxial electric field, we calculated the $\mathrm{FE}$ double well at $e=+3.93 \%$ and the result is displayed in Fig. $4 \mathrm{~b}$. In addition, the FE double well gives the energy difference $\left(\Delta E_{\mathrm{h}}\right)$ between the ground-state FE structure and the high-symmetry paraelectric reference, which can be used to estimate the stability of polarization. According to the calculated $\Delta E_{\mathrm{h}}$, we estimated the Curie temperature of the ferroelectricity and the calculated results are plotted in Fig. $4 \mathrm{c}$ (in the calculation, we take the $\Delta E_{\mathrm{h}}$ of $\mathrm{BaTiO}_{3}$ as a reference). The curve shows that the Curie temperature increases as the strain increases. Especially, it can clearly be seen that when the strain exceeds $6 \%$, the calculated temperature is well above room temperature. In Fig. $4 \mathrm{~d}$ illustrates how the band gaps change as the epitaxial strain increases. With the increase in strain from $+1.61 \%$ to $+9.30 \%$, the calculated band gaps increase from $0.42 \mathrm{eV}$ to $1.41 \mathrm{eV}$ ( $0.84 \mathrm{eV}$ to $1.97 \mathrm{eV}$ with the HSE06 functional) which are suitable for absorption in the solar spectrum. In addition, the band gap tenability of the strained $\mathrm{CsSnI}_{3}$ films is about $0.99 \mathrm{eV}$. Although the band gap tunability is smaller than that previously achieved for FE materials doped with inorganic ions in $\mathrm{KNbO}_{3}$ $(2.50 \mathrm{eV}),{ }^{4}$ the tunable band gaps can be compared with other doped halide perovskite $\mathrm{FE}$ materials, such as (cyclohexylammonium) ${ }_{2} \mathrm{PbBr}_{4-4 x} \mathrm{I}_{4 x} \quad(x=0-0.175)$ and $(N$ methylpyrrolidinium $)_{3} \mathrm{Sb}_{2} \mathrm{Cl}_{9-9 x} \mathrm{~B}_{9 x} \quad(x=0-1){ }^{48}$ To obtain a better understanding of the electric structure of the $P m c 2_{1}$-(I) state, the band structures were calculated. The calculated results show that this state has a direct band gap (Fig. S5†).

Finally, when the tensile strain further increases $(e>$ $+9.30 \%$ ), the $P m c 2_{1}$-(II) state is the ground state. Although it has same space group as the $P m c 2_{1}$-(I) phase, their lattice and electric structures are profoundly different. In addition to the difference in the rotation/tilt of the $\mathrm{SnI}_{6}$ octahedral in these two $P m c 2_{1}$ states, their lattice parameters are also significantly different. Generally, in a strained film, as the in-plane lattice constants increase, the out-of-plane lattice constants decrease. However, it is found that, at the phase boundary, there is significant jump for the $c$-lattice $(0.497 \AA$, see Fig. S6†). In addition, the electric polarization value also has an obvious jump at $e=+9.30 \%\left(6.09 \mu \mathrm{C} \mathrm{cm}^{-2}\right)$. To lower the order in the fields, the energy of the phase with larger polarization will be lowered relative to a phase with smaller polarization by $\vec{P} \cdot \vec{E}$. Thus, for a system in the vicinity of the phase boundary, a large electric-elastic response is predicted as the result of the possibility of switching between two phases with quite different polarization, band gaps, and $c$-lattice parameters. In particular, the $P m c 2_{1}$-(II) state just at the phase boundary could be driven by an applied electric field to the $P m c 2_{1}$-(I) state with a downward jump in the $c$-lattice. In addition, the $P m c 2_{1}$-(II) state could also be driven into the $P m c 2_{1}$-(I) state by an applied uniaxial compressive stress with an upward jump in polarization.

To obtain a better understanding of these results, we next calculated the atom-resolved density of states for each state using the PBEsol method (Fig. 5). In these four phases, we find that the bottom of the conduction band is primary Sn-5p states, whereas the top of the valance band consists primarily of an antibonding combination of Sn-5s and I-5p states for $P 4 \mathrm{bm}$, $P n m a$, and $P m c 2_{1}$-(I) phases which is consistent with a previous study. ${ }^{28}$ Furthermore, there is hybridization among the Sn-5s, 

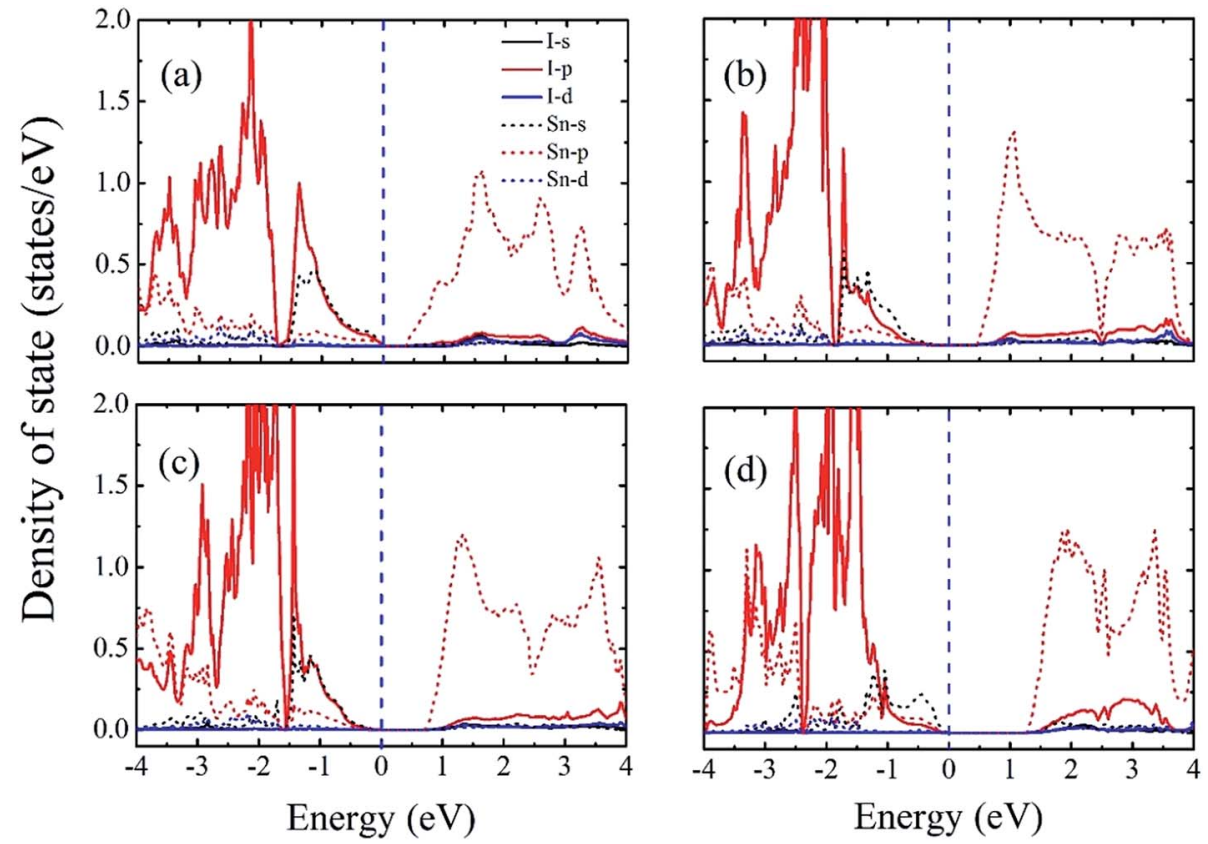

Fig. 5 Atom-resolved density of states for (a) P4bm, (b) Pnma, (c) Pmc2 ${ }_{1}$-(I), and (d) Pmc2 ${ }_{1}$-(II) phases computed using the PBEsol method. The Fermi level is set at $0 \mathrm{eV}$ and is indicated by the blue dashed line.

Sn-5p, and I-5p states throughout the valence and conduction bands. This indicates that the epitaxial strain onhase with smaller hase with smaller films does not modify the orbital character at the band edges for the P4bm, Pnma, and $P m c 2_{1}-(\mathrm{I})$ phases, but instead affects the band gap through structural changes. However, for the Pmc2 ${ }_{1}$-(II) phase, we find that the top of the CBM primarily consists of Sn-5s states. This may be related with the increase in lattice parameters of the $P m c 2_{1}$-(II) phase which can weaken the hybridization between Sn-5s and I$5 p$ below the Fermi energy. Finally, we find that the band gap, broken inversion system, and strongly hybridized Sn-5s and I-5p states enable $P 4 \mathrm{bm}$ (indirect band gap) and $P m c 2_{1}$-(I) (direct band gap) to have a direct p-p optical transition. ${ }^{49}$

\section{Conclusion}

In summary, we have comprehensively investigated the effects of biaxial strain and the accompanying structural distortions on the energy landscapes, electric polarization, and band gaps of films of the perovskite photovoltaic material $\mathrm{CsSnI}_{3}$ with firstprinciple calculations. It is found that both compressive and tensile strain can drive the non-polar phase (Pnma) to the polar phase ( $P 4 \mathrm{bm}$ and $\left.\mathrm{Pmc2}_{1}-(\mathrm{I})\right)$. Notably, our calculations show that the FE polarization is up to several $\mu \mathrm{C} \mathrm{cm}^{-2}$ and that the polarization is considerably enhanced compared with previous studies. Unlike the nonpolar phase, the electric polarization present in these FE phases can effectively separate the photoexcited carriers, leading to novel FE photovoltaic materials with potentially enhanced energy conversion efficiency. Moreover, we show that compressive strain can induce a direct-indirect band gap transformation accompanied with a Pnma to P4bm transition. The $P m c 2_{1}$-(I) phase has a suitable direct band for visible light-harvesting and its band gaps can be altered by several tenths of an electronvolt under epitaxial tensile strain. In addition, we also predict that there is an electric-elastic response in the vicinity of the phase boundary of the $P m c 2_{1}$-(I) and $P m c 2_{1}$-(II) phases. It is expected that all-inorganic strained $\mathrm{CsSnI}_{3}$ films, which possess suitable band gap and large electric polarization, can be used as light-harvesting materials and epitaxial strain can be used as a new route to develop semiconductor FE materials that can promote the desirable separation of photo-excited carriers in the less-explored inorganic perovskite halide materials.

\section{Conflicts of interest}

There are no conflicts to declare.

\section{Acknowledgements}

This study has been supported by the National Natural Science Foundation of China though Grant No. 11604113. We also thank the High Performance Computing Center of Nanjing University for part of the numerical calculations.

\section{References}

1 A. Kojima, K. Teshima, Y. Shirai and T. Miyasaka, J. Am. Chem. Soc., 2009, 131, 6050-6051.

2 M. A. Green, A. Ho-Baillie and H. J. Snaith, Nat. Photonics, 2014, 8, 506-514.

3 K. T. Butler, J. M. Frost and A. Walsh, Energy Environ. Sci., 2015, 8, 838-848. 
4 I. Grinberg, D. V. West, M. Torres, G. Gou, D. M. Stein, L. Wu, G. Chen, E. M. Gallo, A. R. Akbashev, P. K. Davies, J. E. Spanier and A. M. Rappe, Nature, 2013, 503, 509-512.

5 J. E. Spanier, V. M. Fridkin, A. M. Rappe, A. R. Akbashev, A. Polemi, Y. Qi, Z. Gu, S. M. Young, C. J. Hawley, D. Imbrenda, G. Xiao, A. L. Bennett-Jackson and C. L. Johnson, Nat. Photonics, 2016, 10, 611-616.

6 R. Nechache, C. Harnagea, S. Li, L. Cardenas, W. Huang, J. Chakrabartty and F. Rosei, Nat. Photonics, 2015, 9, 61-67.

7 H. Borkar, V. Rao, M. Tomar, V. Gupta, J. F. Scott and A. Kumar, RSC Adv., 2017, 7, 12842-12855.

8 W. Huang, C. Harnagea, D. Benetti, M. Chaker, F. Rosei and R. Nechache, J. Mater. Chem. A, 2017, 5, 10355-10364.

9 J. Kreisel, M. Alexe and P. A. Thomas, Nat. Mater., 2012, 11, 260.

10 J. He, C. Franchini and J. M. Rondinelli, Chem. Mater., 2017, 29, 2445-2451.

11 Z. Fan, J. Xiao, K. Sun, L. Chen, Y. Hu, J. Ouyang, K. P. Ong, K. Zeng and J. Wang, J. Phys. Chem. Lett., 2015, 6, 1155-1161. 12 D. P. McMeekin, G. Sadoughi, W. Rehman, G. E. Eperon, M. Saliba, M. T. Hörantner, A. Haghighirad, N. Sakai, L. Korte, B. Rech, M. B. Johnston, L. M. Herz and H. J. Snaith, Science, 2016, 351, 151-155.

13 J. Burschka, N. Pellet, S.-J. Moon, R. Humphry-Baker, P. Gao, M. K. Nazeeruddin and M. Gratzel, Nature, 2013, 499, 316319.

14 S. Liu, F. Zheng, N. Z. Koocher, H. Takenaka, F. Wang and A. M. Rappe, J. Phys. Chem. Lett., 2015, 6, 693-699.

15 W.-J. Yin, J.-H. Yang, J. Kang, Y. Yan and S.-H. Wei, J. Mater. Chem. A, 2015, 3, 8926-8942.

16 A. Stroppa, C. Quarti, F. De Angelis and S. Picozzi, J. Phys. Chem. Lett., 2015, 6, 2223-2231.

17 J. M. Frost, K. T. Butler, F. Brivio, C. H. Hendon, M. van Schilfgaarde and A. Walsh, Nano Lett., 2014, 14, 2584-2590.

18 G. Sharada, P. Mahale, B. P. Kore, S. Mukherjee, M. S. Pavan, C. De, S. Ghara, A. Sundaresan, A. Pandey, T. N. Guru Row and D. D. Sarma, J. Phys. Chem. Lett., 2016, 7, 2412-2419.

19 Y.-Q. Zhao, B. Liu, Z.-L. Yu, J. Ma, W. Qiang, P.-B. He and M.-Q. Cai, J. Mater. Chem. C, 2017, 5, 5356-5364.

20 R. J. Sutton, G. E. Eperon, L. Miranda, E. S. Parrott, B. A. Kamino, J. B. Patel, M. T. Hörantner, M. B. Johnston, A. A. Haghighirad, D. T. Moore and H. J. Snaith, Adv. Energy Mater., 2016, 6, 1502458.

21 C. Yi, J. Luo, S. Meloni, A. Boziki, N. Ashari-Astani, C. Gratzel, S. M. Zakeeruddin, U. Rothlisberger and M. Gratzel, Energy Environ. Sci., 2016, 9, 656-662.

22 M. Kulbak, D. Cahen and G. Hodes, J. Phys. Chem. Lett., 2015, 6, 2452-2456.

23 Z. Li, M. Yang, J.-S. Park, S.-H. Wei, J. J. Berry and K. Zhu, Chem. Mater., 2016, 28, 284-292.
24 Y. S. Oh, X. Luo, F.-T. Huang, Y. Wang and S.-W. Cheong, Nat. Mater., 2015, 14, 407-413.

25 M. J. Pitcher, P. Mandal, M. S. Dyer, J. Alaria, P. Borisov, H. Niu, J. B. Claridge and M. J. Rosseinsky, Science, 2015, 347, 420-424.

26 N. A. Benedek and C. J. Fennie, Phys. Rev. Lett., 2011, 106, 107204.

27 G. Song, G. Li, B. Gao, F. Liang and J. Zhang, J. Alloys Compd., 2017, 690, 923-929.

28 G. Gou, J. Young, X. Liu and J. M. Rondinelli, Inorg. Chem., 2017, 56, 26-32.

29 I. Chung, B. Lee, J. He, R. P. H. Chang and M. G. Kanatzidis, Nature, 2012, 485, 486-489.

30 I. Chung, J.-H. Song, J. Im, J. Androulakis, C. D. Malliakas, H. Li, A. J. Freeman, J. T. Kenney and M. G. Kanatzidis, J. Am. Chem. Soc., 2012, 134, 8579-8587.

31 K. P. Marshall, M. Walker, R. I. Walton and R. A. Hatton, Nat. Energy, 2016, 1, 16178.

32 J. P. Perdew, A. Ruzsinszky, G. I. Csonka, O. A. Vydrov, G. E. Scuseria, L. A. Constantin, X. Zhou and K. Burke, Phys. Rev. Lett., 2008, 100, 136406.

33 G. Kresse and J. Hafner, Phys. Rev. B: Condens. Matter Mater. Phys., 1993, 47, 558-561.

34 P. E. Blöchl, Phys. Rev. B: Condens. Matter Mater. Phys., 1994, 50, 17953-17979.

35 C. Grote and R. F. Berger, J. Phys. Chem. C, 2015, 119, 2283222837.

36 R. D. King-Smith and D. Vanderbilt, Phys. Rev. B: Condens. Matter Mater. Phys., 1994, 49, 5828-5844.

37 H. T. Stokes and D. M. Hatch, J. Appl. Crystallogr., 2005, 38, 237-238.

38 J. Heyd, G. E. Scuseria and M. Ernzerhof, J. Chem. Phys., 2003, 118, 8207-8215.

39 K. Momma and F. Izumi, J. Appl. Crystallogr., 2011, 44, 12721276.

40 S.-D. Guo and J.-L. Wang, RSC Adv., 2016, 6, 101552-101559. 41 A. Glazer, Acta Crystallogr., 1972, 28, 3384-3392.

42 E. J. Baerends, O. V. Gritsenko and R. van Meer, Phys. Chem. Chem. Phys., 2013, 15, 16408-16425.

43 L. Yu and A. Zunger, Phys. Rev. Lett., 2012, 108, 068701.

44 S. Liu, Y. Kim, L. Z. Tan and A. M. Rappe, Nano Lett., 2016, 16, 1663-1668.

45 S. Chadov, X. Qi, J. Kübler, G. H. Fecher, C. Felser and S. C. Zhang, Nat. Mater., 2010, 9, 541-545.

46 H. Tian, A.-J. Mao, H. J. Zhao, Y. Cui, H. Li and X.-Y. Kuang, Phys. Chem. Chem. Phys., 2016, 18, 7680-7687.

47 Y. Yang, W. Ren, M. Stengel, X. H. Yan and L. Bellaiche, Phys. Rev. Lett., 2012, 109, 057602.

48 C. Ji, Z. Sun, A. Zeb, S. Liu, J. Zhang, M. Hong and J. Luo, J. Phys. Chem. Lett., 2017, 8, 2012-2018.

49 W.-J. Yin, T. Shi and Y. Yan, Adv. Mater., 2014, 26, 4653-4658. 